\title{
Efecto de la temperatura y concentración de panela en la osmodeshidratación de maca asistido por ultrasonido
}

\section{Effect of temperature and panela concentration on ultrasound-assisted maca osmodehydration}

\begin{abstract}
Rodolfo M. Vegas ${ }^{1} \&$ Yuli R. Baltazar ${ }^{1}$
${ }^{1}$ Universidad Nacional de Trujillo (UNT), Escuela de Ingeniería Agroindustrial-Filial Huamachuco. Perú Correoelectrónico:rvegas@unitru.edu.pe
\end{abstract}

\section{Resumen}

La presente investigación tuvo como objetivo determinar los valores óptimos de temperatura $\left(30-60^{\circ} \mathrm{C}\right)$ y concentración de panela $(30-60 \% \mathrm{p} / \mathrm{p})$, en la cinética de osmodeshidratación de cubos de maca variedad amarilla (Lepidium meyenii) asistido por ultrasonido $(40 \mathrm{kHz})$. Se estableció la metodología de superficie de respuesta con un diseño factorial estadístico $2 \mathrm{k}+2(\mathrm{k})+3$ puntos centrales, sometiendo las muesttras a tratamiento con ultrasonido $\mathrm{y}$ un testigo. Los resultados mostraron una pérdida máxima de agua de 53.57 y $24.56 \%$ en los tratamientos con ultrasonido y sin movimiento, mientras que la máxima ganancia de sólidos fue de 18.61 y 9.01 $\%$ respectivamente. En cuanto a los coeficientes de difusividad, se obtuvo valores entre $2.51-4.74 \times 10^{-10} \mathrm{~m}^{2} / \mathrm{s}$ para el agua y $1.16-2.20 \times 10^{-10}$ $\mathrm{m}^{2} / \mathrm{s}$ para los sólidos en el tratamiento con ultrasonido y entre $1.10-2.35$ x $10^{-10} \mathrm{~m}^{2} / \mathrm{s}$ para el agua y $0.90-1.70 \times 10^{-10} \mathrm{~m}^{2} / \mathrm{s}$ para los sólidos en el tratamiento testigo. El análisis de varianza mostró un efecto significativo $\left(\mathrm{p}<0.05 \mathrm{y} \mathrm{R}^{2} \approx 1\right)$ por parte de las variables estudiadas de manera lineal, cuadrática e interaccionadas. Como conclusión se tiene que los máximos valores de coeficiente de difusividad tanto para el agua como en sólidos en el tratamiento con ultrasonido se obtuvo en concentraciones de panela entre $54-61 \% \mathrm{p} / \mathrm{p}$ y temperatura $48-54{ }^{\circ} \mathrm{C}$ y para el tratamiento testigo a concentraciones de panela de $50-56 \% \mathrm{p} / \mathrm{p}$ y temperatura entre $46-52{ }^{\circ} \mathrm{C}$.

Palabras clave: Osmodeshidratación, difusividad, panela, ultrasonido, maca.

\begin{abstract}
The present investigation aimed to determine the optimal values of temperature $\left(30-60{ }^{\circ} \mathrm{C}\right)$ and panela concentration $(30-60 \% \mathrm{w} / \mathrm{w})$, on the osmodehydration kinetics of cubes of yellow variety maca (Lepidium meyenii) assisted by ultrasound. $(40 \mathrm{kHz})$. The response surface methodology was established with a statistical factorial design $2 \mathrm{k}+2(\mathrm{k})$ +3 central points was established, subjecting the samples to ultrasound treatment and a control. The results showed a maximum water loss of 53.57 and $24.56 \%$ in the treatments with ultrasound and without movement,
\end{abstract}


while the maximum gain in solids was 18.61 and $9.01 \%$ respectively. Regarding the diffusivity coefficients, values between $2.51-4.74 \mathrm{x}$ $10^{-10} \mathrm{~m}^{2} / \mathrm{s}$ were obtained for water and $1.16-2.20 \times 10^{-10} \mathrm{~m}^{2} / \mathrm{s}$ for solids in the ultrasound treatment and between $1.10-2.35 \times 10^{-10} \mathrm{~m}^{2} / \mathrm{s}$ for water and $0.90-1.70 \times 10^{-10} \mathrm{~m}^{2} / \mathrm{s}$ for solids in the control treatment. The analysis of variance showed a significant effect $\left(\mathrm{p}<0.05\right.$ and $\left.\mathrm{R}^{2} \approx 1\right)$ on the part of the variables studied in a linear, quadratic and interacting manner. As a conclusion we have that the maximum values of diffusivity coefficient for both water and solids in the ultrasound treatment were obtained in panela concentrations between 54-61\% w/w and temperature $48-54{ }^{\circ} \mathrm{C}$ and for the control treatment at panela concentrations of $50-56 \%$ $\mathrm{w} / \mathrm{w}$ and temperature between $46-52^{\circ} \mathrm{C}$.

Keywords: Osmodehydration, diffusivity, panela, ultrasound, maca.

\section{Introducción}

La maca (Lepidium meyenii) crece en los andes del Perú a 4000 y 4500 msnm. Hay diferentes variedades de maca según tamaño y color del hipocótilo como son amarillo, negro y rojo (Valerio y Gonzales, 2005). Se ha demostrado que la raíz de la maca tiene un efecto promotor de fertilidad femenina y masculina, mejora también la función sexual, inmunidad, memoria, proporciona vitalidad y energía, tiene capacidad antioxidante, antinflamatoria, así como también es un atenuante a problemas de próstata y osteoporosis (Gonzales, 2006). Además, la maca es una importante fuente de minerales (fósforo, sodio, hierro, potasio, calcio) y vitaminas (B1, B2, B6, B12 y ácido ascórbico). Estudios nutricionales indican que la raíz de la maca contiene también aminoácidos esenciales, proteínas, carbohidratos y fibra (Obregón, 1998).

Barbosa y Vega (2000) establecen a la osmodeshidratación como un método que permite disminuir el contenido de humedad y aumentar los sólidos solubles en las frutas. Y gracias a la eliminación de agua de la fruta, Suca y Suca (2010) consideran se evita el pardeamiento enzimático, ya que al disminuir la actividad del agua disminuye la actividad de la enzima, así como la acción por parte de los microorganismos.

El ultrasonido ha sido usado para mejorar los procesos alimenticios por su bajo impacto en el ambiente. El ultrasonido aumenta la transferencia de masa y calor por lo tanto disminuye el tiempo de proceso, manteniendo las propiedades nutricionales y sensoriales del alimento (Dane, 2015; FAO, 1987).

El objetivo del presente trabajo fue determinar el coeficiente de difusividad media efectiva y los parámetros óptimos de temperatura y concentración de panela en la osmodeshidratación de maca (Lepidium meyenii) por ultrasonido. 


\section{Materiales y Métodos}

\section{Materia prima}

Se empleó maca (Lepidium meyenii) variedad amarilla, procedente de la localidad de Jaulabamba, provincia de Santiago de Chuco (Departamento La Libertad). Se retiró la cáscara y se cortaron cubos de $1.0 \times 1.0 \times 1.0 \mathrm{~cm}$.

\section{Análisis fisicoquímico de la maca}

Para la determinación de las características fisicoquímicas, se determinó el contenido de humedad y sólidos (ISO 638: 2008), contenido de cenizas (ISO 776:1982), sólidos solubles ( ${ }^{\circ}$ Brix) (NMX-F-103-1982), azúcares reductores (NMX-F-312-1978) y capacidad antioxidante (Brand-Willams et al., 1995).

\section{Preparación de la solución osmótica (DO)} osmótico.

Se preparó una solución hipertónica $(30-60 \%$ p/p), usando panela como soluto

\section{Deshidratación osmótica}

Los cubos de maca se sumergieron en la solución hipertónica en recipientes de vidrio para cada tratamiento, la relación maca - panela fue de 1:10 por un tiempo de 7 horas que es apto para llevar el proceso de osmodeshidratación (Azuara et al., 2002). Estos recipientes se ubicaron en el equipo de ultrasonido (BRANSONIC, modelo 8510E-DTH), con panel de control temperatura, tiempo y frecuencia $(40 \mathrm{kHz})$ y cada tratamiento fue retirado del ultrasonido según el tiempo correspondiente

Posteriormente las muestras se dispusieron sobre papel absorbente para retirar el exceso de jarabe impregnado.

\section{Pesado y secado}

Para analizar la cinética de DO, fue necesario pesar la maca antes de secarlos, para cuantificar los sólidos absorbidos al interior de la muestra. Se procedió a secar las muestras a $105^{\circ} \mathrm{C}$ por aproximadamente 24 horas hasta alcanzar peso constante. Una vez que alcancen peso constante se retiró de la estufa y se enfrió en campanas de desecación $\mathrm{y}$ fueron pesadas.

\section{Cálculo del coeficiente de difusividad}

Para el cálculo de difusividad efectiva para el agua y sólidos en una geometría cúbica, se empleó las ecuaciones (1) y (2) propuesta por Azuara et al. (1998):

$$
\begin{aligned}
& \left.D_{t, w}=\frac{\pi}{4 . t^{\frac{1}{3}}}\left[\left(\frac{S_{1} L^{3}}{1+S_{1} t}\right)\left(\frac{W L_{\infty}^{\bmod }}{W L_{\infty}^{\exp }}\right)\right]^{2 / 3} \ldots \ldots \ldots \ldots \ldots \ldots \ldots \ldots \ldots \text { (ec. } 1\right)
\end{aligned}
$$

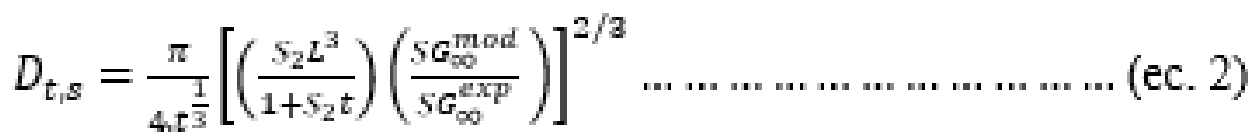


Donde $\mathrm{D}_{\mathrm{e}, \mathrm{w}} \quad \mathrm{y} \quad \mathrm{D}_{\mathrm{e}, \mathrm{s}}$ representan los coeficientes de difusión aparente para el agua y sólidos respectivamente al tiempo t; $W L_{\infty}^{\bmod }$ y $S G_{\infty}^{\bmod }$ son los valores de equilibrio para la pérdida de agua y ganancia de sólidos; $S_{1}$ y $S_{2}$ son las constantes relacionadas con la pérdida de agua y ganancia de sólidos respectivamente; $W L_{\infty}{ }^{\text {mod }}$ y $S G_{\infty}$ mod son los valores de equilibrio obtenidos de la experimentación; y L representa la longitud de arista del cubo. El cálculo del coeficiente de difusividad promedio se determinó a través de la ecuación 3 :

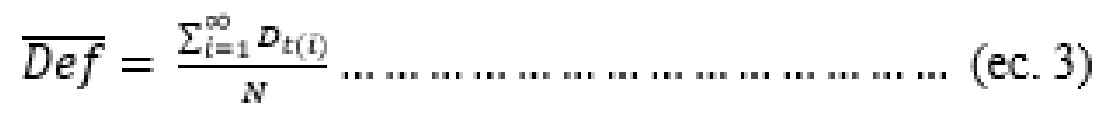

$(\text { Def })^{-}=$Difusividad efectiva media $\left(\mathrm{m}^{2} / \mathrm{s}\right) ; \mathrm{D}_{\mathrm{t}(\mathrm{i})}=$ difusividad efectiva para cada tiempo $\left(\mathrm{m}^{2} / \mathrm{s}\right) ; \mathrm{N}=$ Número de puntos experimentales utilizados para el cálculo.

\section{Análisis estadístico}

Se estableció un diseño compuesto central rotable (DCCR) mediante un planeamiento factorial completo $2^{\mathrm{k}}\left(2^{2}\right)$ puntos factoriales, 4 puntos axiales y 3 repeticiones en el punto central, totalizando 11 ensayos (según Tabla 2). K, representa el número de variables independientes $(\mathrm{K}=2)$ tiempo y concentración de panela.

\section{Resultados y discusión}

\section{Caracterización fisicoquímica de la maca}

En la Tabla 1 se reporta la composición fisicoquímica de los cubos de maca Lepidium meyenii variedad amarilla. El contenido de humedad y de cenizas obtuvieron valores cercanos al reportado por el Centro Nacional de Alimentación y Nutrición (2017) con un valor de $79.8 \%$ y $2.5 \mathrm{~g} / 100 \mathrm{~g}$ respectivamente.

Los sólidos solubles de la maca fueron de $6.67 \pm 0.58^{\circ}$ Brix, este valor es menor comparado con otras raíces como yacón (10.3 ${ }^{\circ}$ Brix) (Bocanegra y Espinosa, 2013). Dentro de los sólidos solubles se incluyen ciertas proteínas, minerales, ácidos orgánicos y carbohidratos, siendo indicadores del grado de dulzor (Damodaran y Parkin, 2017). En tanto los valores de azúcares reductores fue de $2.50 \pm 0.04 \%$.

La capacidad antioxidante fue de $19.81 \pm 1.11 \mathrm{mg} / 100 \mathrm{~g}$ (calculado como \% de captación de radicales libres del 2,2 difenil-1-picrilhidrazilo, DPPH). Yao et al. (2012) demostraron que los polisacáridos de la maca tienen una alta actividad antioxidante y podrían constituir fuente de compuestos bioactivos. 
Tabla 1

Caracterización fisicoquímica de la maca (Lepidium meyenii) variedad amarilla

\begin{tabular}{lcc}
\hline \multicolumn{1}{c}{ Componente } & Unidad de medida & Cantidad \\
\hline Humedad & $\%$ & $79.31 \pm 0.36$ \\
Cenizas & $\mathrm{g} / 100 \mathrm{~g}$ & $2.41 \pm 0.21$ \\
Sólidos Solubles & ${ }^{\circ} \mathrm{Brix}$ & $6.67 \pm 0.58$ \\
Azúcares reductores & $\%$ & $2.50 \pm 0.04$ \\
Capacidad antioxidante* & $\%$ & $19.81 \pm 1.11$ \\
\hline "Expresado como \% de captación de radicales libres por el 2,2 -difenil-1- \\
picrillidrazilo (DPPH) en forma de radical libre.
\end{tabular}

\section{Cinética de osmodeshidratación}

En las Figuras 1 y 2 se puede observar la pérdida de agua (\% WL) y ganancia de solutos (\% SG) en la deshidratación osmótica de cubos de maca tanto en condiciones testigo como en ultrasonido. En ambas se observa una tendencia logarítmica. Se ha considerado que la osmodeshidratación llega a retirar entre un $40-65 \%$ de agua presente en la muestra fresca (Suca, 2008; Karathanos et al.,1995) esto dependiendo del tipo de alimento.

Durante las dos primeras horas de tratamiento se observa la mayor pérdida de agua y ganancia de sólidos. Raoult y Wack (1992) consideran que la pérdida de agua y ganancia de sólidos es alta al iniciar el proceso. Según datos experimentales $\left(45^{\circ} \mathrm{C}\right.$ y $60 \%$ p/p por 7 horas) se logró eliminar el $53.57 \%$ y $24.56 \%$ de agua en los tratamientos con ultrasonido y testigo respectivamente; en tanto que la ganancia de sólidos fue de $18.61 \%$ con ultrasonido y $9.01 \%$ en el tratamiento testigo.

\section{Figura 1}

Cinética de pérdida de agua (\% WL) en la deshidratación osmótica de cubos de maca.

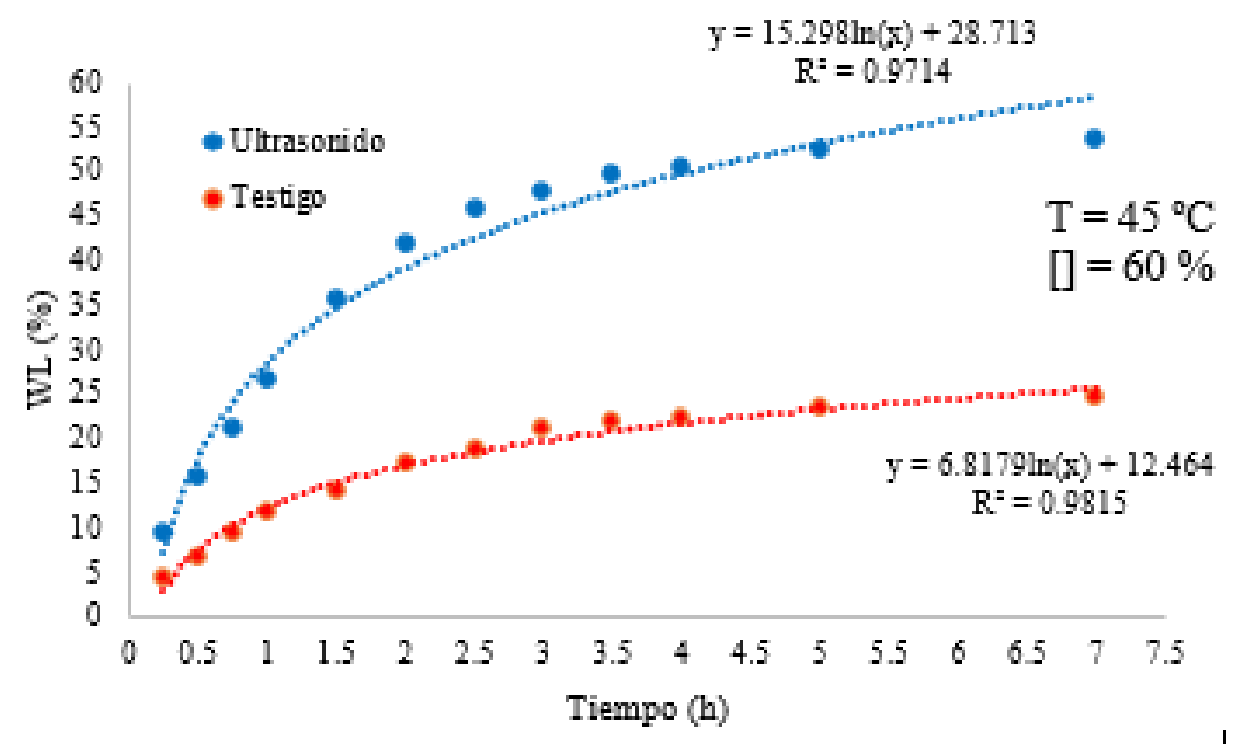




\section{Figura 2}

Cinética de ganancia de solutos (\% SG) en la deshidratación osmótica de maca en cubos.

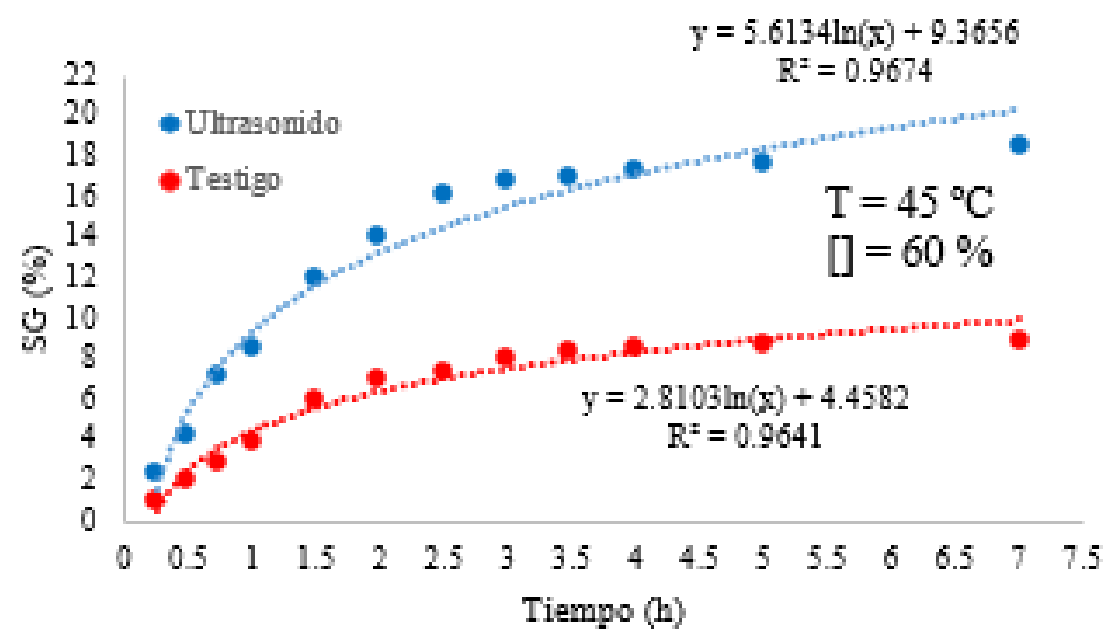

\section{Cálculo de la difusividad efectiva}

En las Tablas 2 y 3 se establecen los coeficientes de difusividad para el agua y sólidos en los ensayos con ultrasonido y testigo respectivamente.

Según Nahimana et al. (2011) los coeficientes de difusividad efectiva en la osmodeshidratación de vegetales y frutas varía entre $1.39-42.3$ y $0.42-36.1$ x $10^{-10} \mathrm{~m}^{2} / \mathrm{s}$ para el agua y soluto osmótico respectivamente. Estas variaciones se deben a las diferencias en la estructura y composición del alimento, así como a las condiciones de proceso (tiempo, temperatura y concentración del medio osmótico). Además, gran parte de frutas y verduras tienen diversas resistencias de transferencia de masa debido a la diferencia de sus membranas y composición de sus paredes celulares.

En las Tablas 2 y 3 se muestra el promedio de los coeficientes de difusividad, tanto para el agua y soluto en ensayos con ultrasonido y sin el mismo (sin movimiento). En los tratamientos con ultrasonido $(40 \mathrm{kHz})$ los coeficientes de difusividad tuvieron una variación de $2.514-4.741$ y de $1.164-2.208 \times 10^{-10} \mathrm{~m}^{2} / \mathrm{s}$ para el agua y soluto respectivamente. Mientras que el tratamiento testigo (sin movimiento) los coeficientes variaron entre $1.101-2.350 \times 10^{-10} \mathrm{~m}^{2} / \mathrm{s}$ y $0.901-1.702 \times 10^{-10} \mathrm{~m}^{2} / \mathrm{s}$ para el agua $\mathrm{y}$ sólidos respectivamente hallándose dentro de los intervalos reportados por Nihamana et al. (2011).

Dermesonlouoglou et al. (2007) consideran que el coeficiente de difusividad (De) o también denominado flujo difusivo es una medida del contenido de masa que atraviesa una unidad de área en un determinado tiempo. En deshidratación osmótica de camote Rodríguez (2019) reporta valores entre $3.223-6.052 \times 10^{-10} \mathrm{~m}^{2} / \mathrm{s}$ y entre $1.594-2.650 \mathrm{x}$ $10^{-10} \mathrm{~m}^{2} / \mathrm{s}$ para el agua y sólidos con el uso de ultrasonido $\left(40 \mathrm{kHz}\right.$, temperatura $45^{\circ} \mathrm{C} \mathrm{y}$ concentración de panela $60 \% \mathrm{p} / \mathrm{p}$ ). En tanto en el tratamiento testigo reportó valores de coeficiente de difusividad para el agua de $1.504-2.709 \times 10^{-10} \mathrm{~m}^{2} / \mathrm{s}$ y para ganancia de sólidos $1.079-1.811 \times 10^{-10} \mathrm{~m}^{2} / \mathrm{s}$. 
Tabla 2

Valores de difusividad determinados por el modelo de Azuara para la osmodeshidratación de cubos de maca asistido con ultrasonido $(40 \mathrm{kHz})$

\begin{tabular}{|c|c|c|c|c|}
\hline \multirow{2}{*}{ Ensayos } & \multirow{2}{*}{$\begin{array}{c}\text { Temperatura } \\
\left.\text { ( }{ }^{\circ} \mathrm{C}\right)\end{array}$} & \multirow{2}{*}{$\begin{array}{c}\text { Concentración } \\
\text { de panela } \\
\text { (\% p/p) }\end{array}$} & \multicolumn{2}{|c|}{$\begin{array}{l}\text { Coeficiente de difusividad } \\
\qquad\left(\mathrm{m}^{2} / \mathrm{s}\right) \times 10^{-10}\end{array}$} \\
\hline & & & Agua $\left(D_{c, w}\right)$ & Sólidos $\left(\mathrm{D}_{c, 1}\right)$ \\
\hline 1 & 34.4 & 34.4 & 1.1011 & 0.9016 \\
\hline 2 & 34.4 & 55.6 & 1.8101 & 1.3217 \\
\hline 3 & 55.6 & 34.4 & 1.1906 & 0.9745 \\
\hline 4 & 55.6 & 55.6 & 2.3017 & 1.6241 \\
\hline 5 & 30 & 45 & 1.2801 & 1.0128 \\
\hline 6 & 60 & 45 & 1.9402 & 1.5034 \\
\hline 7 & 45 & 30 & 1.1423 & 0.9353 \\
\hline 8 & 45 & 60 & 2.3500 & 1.7021 \\
\hline 9 & 45 & 45 & 1.9451 & 1.6126 \\
\hline 10 & 45 & 45 & 1.9503 & 1.6092 \\
\hline 11 & 45 & 45 & 1.9497 & 1.6058 \\
\hline
\end{tabular}

Tabla 3

Valores de difusividad determinados por el modelo de Azuara para el tratamiento de osmodeshidratación de cubos de maca sin movimiento (testigo)

\begin{tabular}{|c|c|c|c|c|}
\hline \multirow[t]{2}{*}{ Ensayos } & \multirow{2}{*}{$\begin{array}{c}\text { Temperatura } \\
\text { ( } \mathrm{C})\end{array}$} & \multirow{2}{*}{$\begin{array}{c}\text { Concentración } \\
\text { de panela } \\
(\% \text { p } / p)\end{array}$} & \multicolumn{2}{|c|}{$\begin{array}{l}\text { Coeficiente de difusividad } \\
\qquad\left(\mathrm{m}^{2} / s\right) \times 10^{-10}\end{array}$} \\
\hline & & & Agua $\left(\mathrm{D}_{\varepsilon, \pi}\right)$ & Sólidos $\left(\mathrm{D}_{c, \mathrm{~s}}\right)$ \\
\hline 1 & 34.4 & 34.4 & 1.1011 & 0.9016 \\
\hline 2 & 34.4 & 55.6 & 1.8101 & 1.3217 \\
\hline 3 & 55.6 & 34.4 & 1.1906 & 0.9745 \\
\hline 4 & 55.6 & 55.6 & 2.3017 & 1.6241 \\
\hline 5 & 30 & 45 & 1.2801 & 1.0128 \\
\hline 6 & 60 & 45 & 1.9402 & 1.5034 \\
\hline 7 & 45 & 30 & 1.1423 & 0.9353 \\
\hline 8 & 45 & 60 & 2.3500 & 1.7021 \\
\hline 9 & 45 & 45 & 1.9451 & 1.6126 \\
\hline 10 & 45 & 45 & 1.9503 & 1.6092 \\
\hline 11 & 45 & 45 & 1.9497 & 1.6058 \\
\hline
\end{tabular}

\section{Análisis estadístico}

Se realizó un análisis de varianza (ANVA) para los coeficientes de difusividad de agua $\left(\mathrm{D}_{\mathrm{e}, \mathrm{w}}\right)$ y soluto $\left(\mathrm{D}_{\mathrm{e}, \mathrm{s}}\right)$ en la deshidratación osmótica de cubos de maca con ultrasonido $(40 \mathrm{kHz})$ y testigo, en donde las variables independientes como se muestra en las Tabla 4,5, 6 y 7 , temperatura $\left({ }^{\circ} \mathrm{C}\right)$ y concentración de panela $(\% \mathrm{p} / \mathrm{p}) \mathrm{de}$ manera lineal (L), cuadrática (Q) y la interacción entre ambas variables (1L x 2L) sobre los coeficientes de difusividad efectiva tanto para la eliminación de agua $\left(\mathrm{D}_{\mathrm{w}}\right)$ y ganancia de 
sólidos $\left(\mathrm{D}_{\mathrm{s}}\right)$ con ultrasonido muestran un efecto significativo de $\mathrm{p}<0.05$ y $\mathrm{R}^{2}$ cercano a 1 .

Tabla 4

Análisis de varianza para $D_{e, W}$ en la deshidratación osmótica de cubos de maca con ultrasonido $(40 \mathrm{kHz})$

\begin{tabular}{lccccc}
\hline \multicolumn{1}{c}{ Factor } & Suma de cuadrados & gl & $\begin{array}{c}\text { Media de } \\
\text { cuadrados }\end{array}$ & Razón F & Valor - p \\
\hline (1)Temperatura $\left({ }^{\circ} \mathrm{C}\right)(\mathrm{L})$ & 0.798771 & 1 & 0.798771 & 188.120 & 0.000037 \\
Temperatura $\left({ }^{\circ} \mathrm{C}\right)(\mathrm{Q})$ & 2.597030 & 1 & 2.597030 & 611.633 & 0.000002 \\
$(2)$ Panela $(\%)(\mathrm{L})$ & 4.500768 & 1 & 4.500768 & 1059.986 & 0.000001 \\
Panela $(\%)(\mathrm{Q})$ & 0.885286 & 1 & 0.885286 & 208.496 & 0.000029 \\
$1 \mathrm{~L} \times 2 \mathrm{~L}$ & 0.542359 & 1 & 0.542359 & 127.732 & 0.000095 \\
Error & 0.021230 & 5 & 0.004246 & & \\
\hline Total suma de cuadrados & 8.697968 & 10 & & & \\
$\mathrm{R}^{2}=0.99756 ; \mathrm{R}_{\text {Aistado }}^{2}=0.99512 ;$ Media de cuadrados residual $=$ \\
0.0042461
\end{tabular}

\section{Tabla 5}

Análisis de varianza para $D_{e, s}$ en la deshidratación osmótica de cubos de maca con ultrasonido $(40 \mathrm{kHz})$

\begin{tabular}{lccccc}
\hline \multicolumn{1}{c}{ Factor } & $\begin{array}{c}\text { Suma de } \\
\text { cuadrados }\end{array}$ & gl & $\begin{array}{c}\text { Media de } \\
\text { cuadrados }\end{array}$ & Razón F & Valor - p \\
\hline (1) Temperatura $\left({ }^{\circ} \mathrm{C}\right)(\mathrm{L})$ & 0.716206 & 1 & 0.716206 & 120.7265 & 0.000109 \\
Temperatura $\left({ }^{\circ} \mathrm{C}\right)(\mathrm{Q})$ & 0.175809 & 1 & 0.175809 & 29.6352 & 0.002840 \\
(2) Panela $(\%)(\mathrm{L})$ & 0.125455 & 1 & 0.125455 & 21.1472 & 0.005848 \\
Panela $(\%)(\mathrm{Q})$ & 0.140621 & 1 & 0.140621 & 23.7037 & 0.004598 \\
$1 \mathrm{~L} \times 2 \mathrm{~L}$ & 0.042932 & 1 & 0.042932 & 7.2368 & 0.043292 \\
Error & 0.029662 & 5 & 0.005932 & & \\
\hline Total suma de cuadrados & 1.159296 & 10 & & & \\
\hline
\end{tabular}

$\mathrm{R}^{2}=0.97441 ; \mathrm{R}_{\text {Ajstado }}=0.94883 ;$ Media de cuadrados residual $=$ 0.0059325

\section{Tabla 6}

Análisis de varianza para $D_{e, w}$ en la deshidratación osmótica de cubos de maca sin movimiento (Testigo).

\begin{tabular}{lccccc}
\hline \multicolumn{1}{c}{ Factor } & $\begin{array}{c}\text { Suma de } \\
\text { cuadrados }\end{array}$ & gl & $\begin{array}{c}\text { Media de } \\
\text { cuadrados }\end{array}$ & Razón F & Valor - p \\
\hline (1) Temperatura $\left({ }^{\circ} \mathrm{C}\right)(\mathrm{L})$ & 0.286802 & 1 & 0.286802 & 49.1686 & 0.000910 \\
Temperatura $\left({ }^{\circ} \mathrm{C}\right)(\mathrm{Q})$ & 0.200446 & 1 & 0.200446 & 34.3640 & 0.002048 \\
(2) Panela (\%p/p)(L) & 1.555857 & 1 & 1.555857 & 266.7324 & 0.000016 \\
Panela $(\% \mathrm{p} / \mathrm{p})(\mathrm{Q})$ & 0.081902 & 1 & 0.081902 & 14.0411 & 0.013333 \\
lL by 2L & 0.040421 & 1 & 0.040421 & 6.9297 & 0.046397 \\
Error & 0.029165 & 5 & 0.005833 & & \\
\hline
\end{tabular}

Total suma de cuadrados $2.138748 \quad 10$

$\mathrm{R}^{L}=0.98636 ; \mathrm{R}_{\text {Ajsido }}^{2}=0.97273$; Media de cuadrados residual

$=0.005833$ 


\section{Tabla 7}

Análisis de varianza para $D_{e, s}$ en la deshidratación osmótica de cubos de maca sin movimiento (Testigo).

\begin{tabular}{|c|c|c|c|c|c|}
\hline Factor & $\begin{array}{l}\text { Suma de } \\
\text { cuadrados }\end{array}$ & gl & $\begin{array}{l}\text { Media de } \\
\text { cuadrados }\end{array}$ & Razón F & Valor - p \\
\hline (1)Temperatura $\left({ }^{\circ} \mathrm{C}\right)(\mathrm{L})$ & 0.142902 & 1 & 0.142902 & 26.8554 & 0.003519 \\
\hline Temperatura $\left({ }^{\circ} \mathrm{C}\right)(\mathrm{Q})$ & 0.217530 & 1 & 0.217530 & 40.8802 & 0.001387 \\
\hline (2)Panela (\%p/p)(L) & 0.580031 & 1 & 0.580031 & 109.0048 & 0.000139 \\
\hline Panela $(\% p / p)(Q)$ & 0.155572 & 1 & 0.155572 & 29.2366 & 0.002925 \\
\hline $1 \mathrm{~L}$ by $2 \mathrm{~L}$ & 0.013168 & 1 & 0.013168 & 2.4746 & 0.176511 \\
\hline Error & 0.026606 & 5 & 0.005321 & & \\
\hline Total suma de cuadrados & 1.052557 & 10 & & & \\
\hline
\end{tabular}

En el diagrama de Pareto (Figura 3, 4, 5 y 6) se visualiza, el efecto de las variables independientes (temperatura y concentración de panela) en el cual se puede observar que el nivel de significancia es mayor a 0.05 y por lo tanto un efecto estadísticamente significativo sobre las variables dependientes (coeficientes de difusividad efectiva).

\section{Figura 3}

Gráfico de Pareto para los coeficientes de difusividad del agua $\left(D_{e, W}\right.$ ) en la deshidratación osmótica de cubos de maca con ultrasonido (40 kHz).

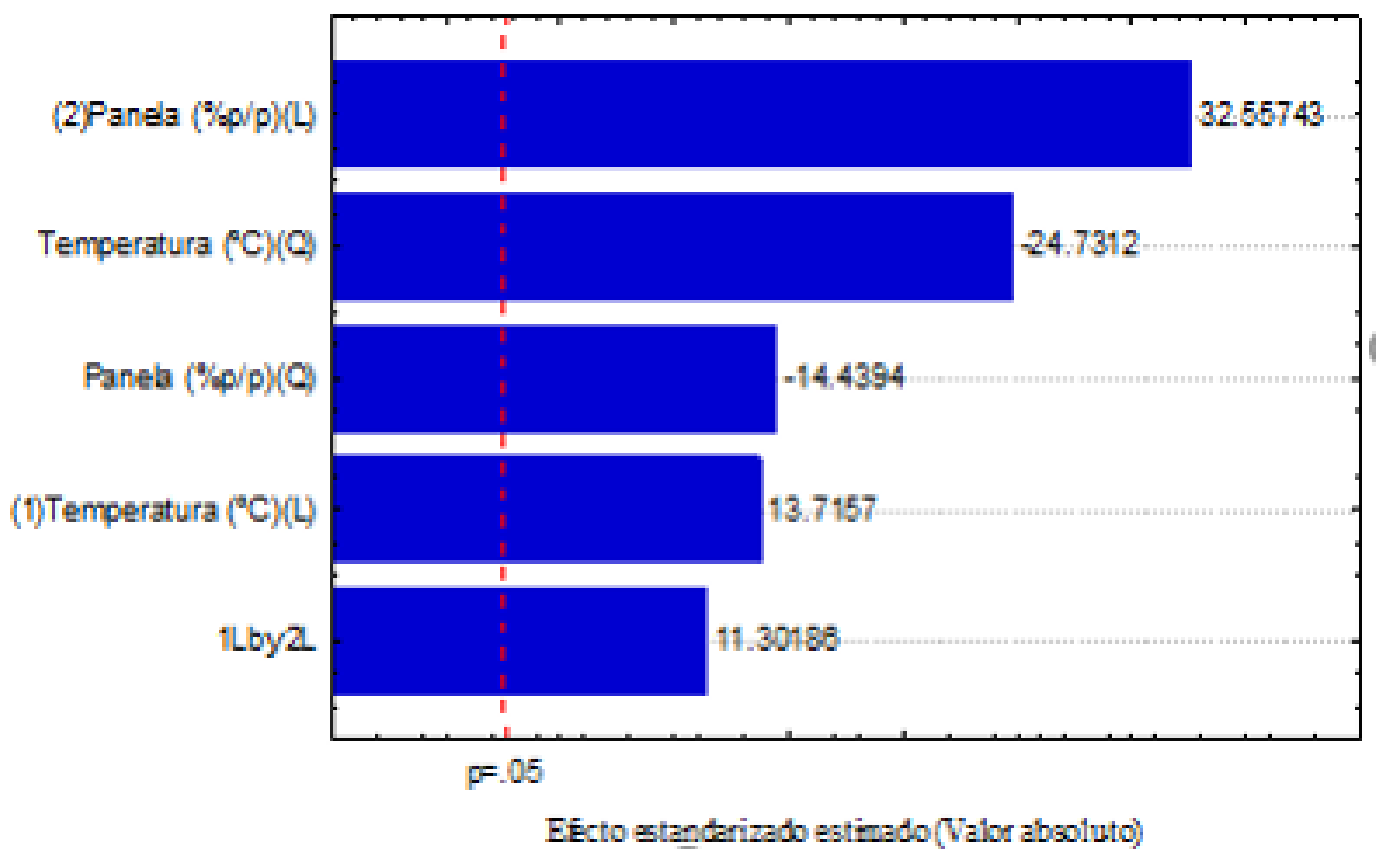




\section{Figura 4}

Gráfico de Pareto para los coeficientes de difusividad de sólidos ( $D_{e, s}$ ) en la deshidratación osmótica de cubos de maca con ultrasonido (40 kHz).

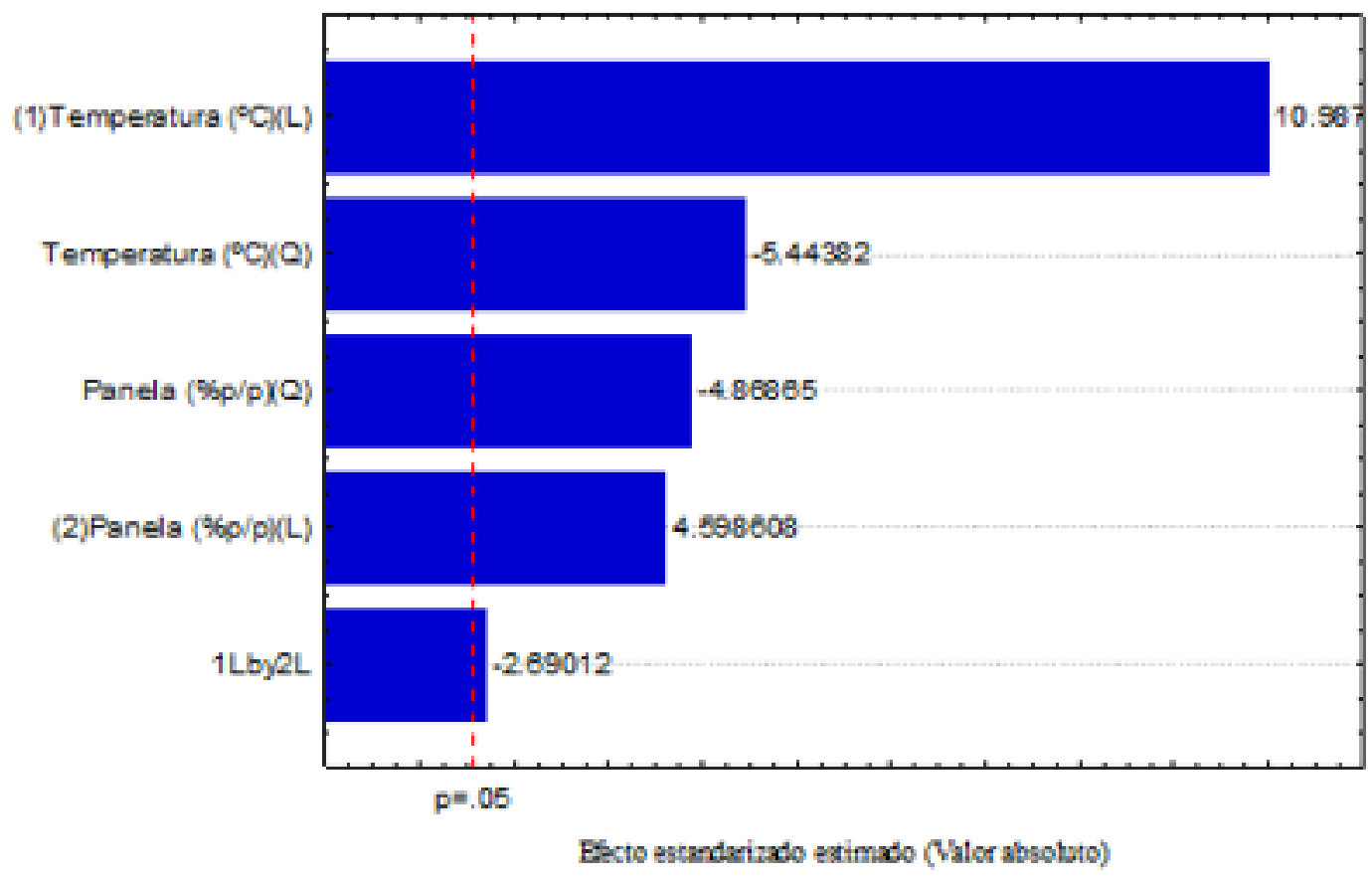

\section{Figura 5}

Gráfico de Pareto para los coeficientes de las variables independientes en la determinación de la difusividad del agua $\left(D_{e, w}\right.$ ) en la osmodeshidratación de cubos de maca (testigo).

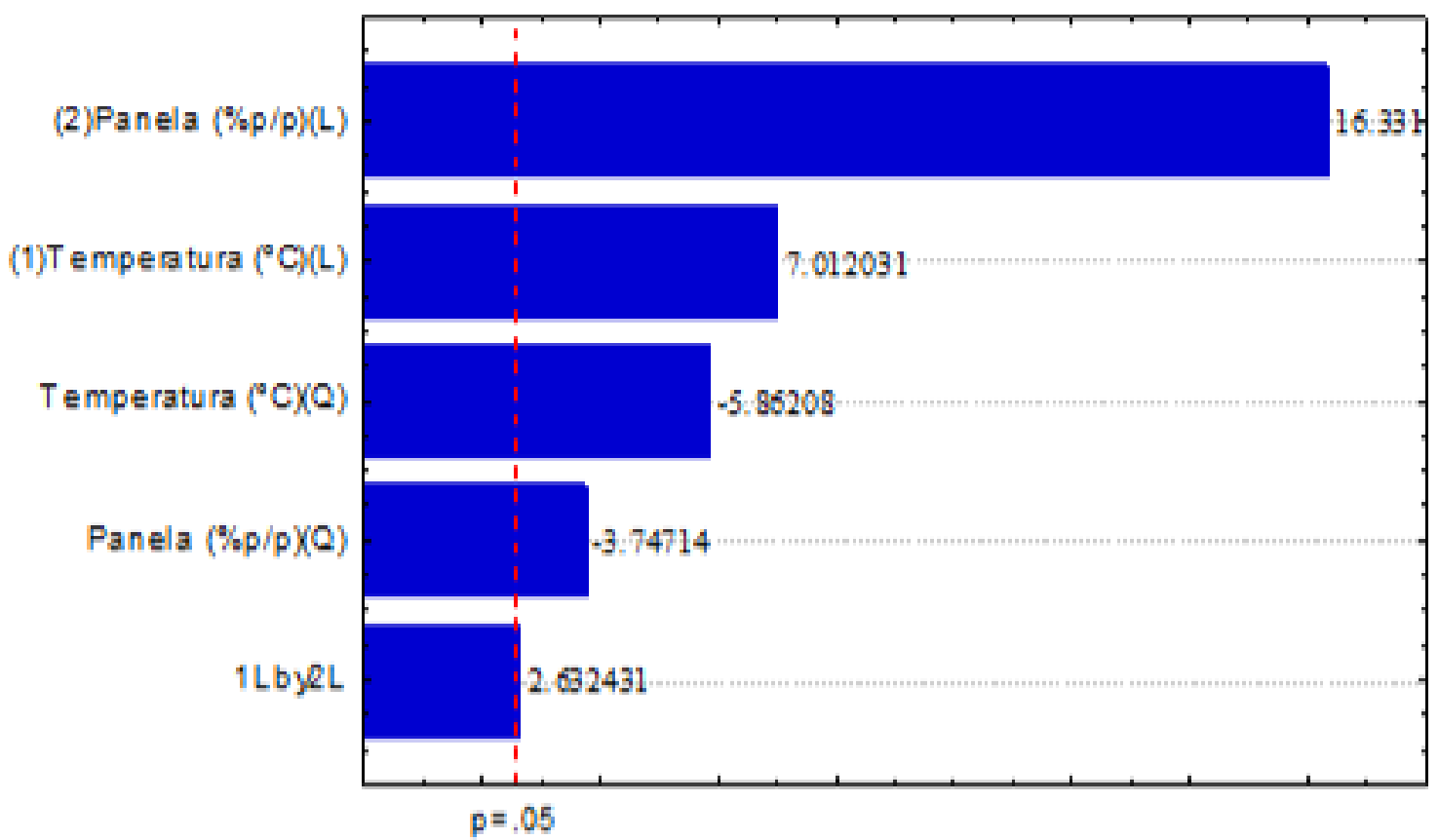

Efecto estandrizado estim ado (Valor absoluto) 


\section{Figura 6}

Gráfico de Pareto para los coeficientes de las variables independientes en la determinación de la difusividad de sólidos $\left(D_{e, s}\right)$ en la osmodeshidratación de cubos de maca (testigo).

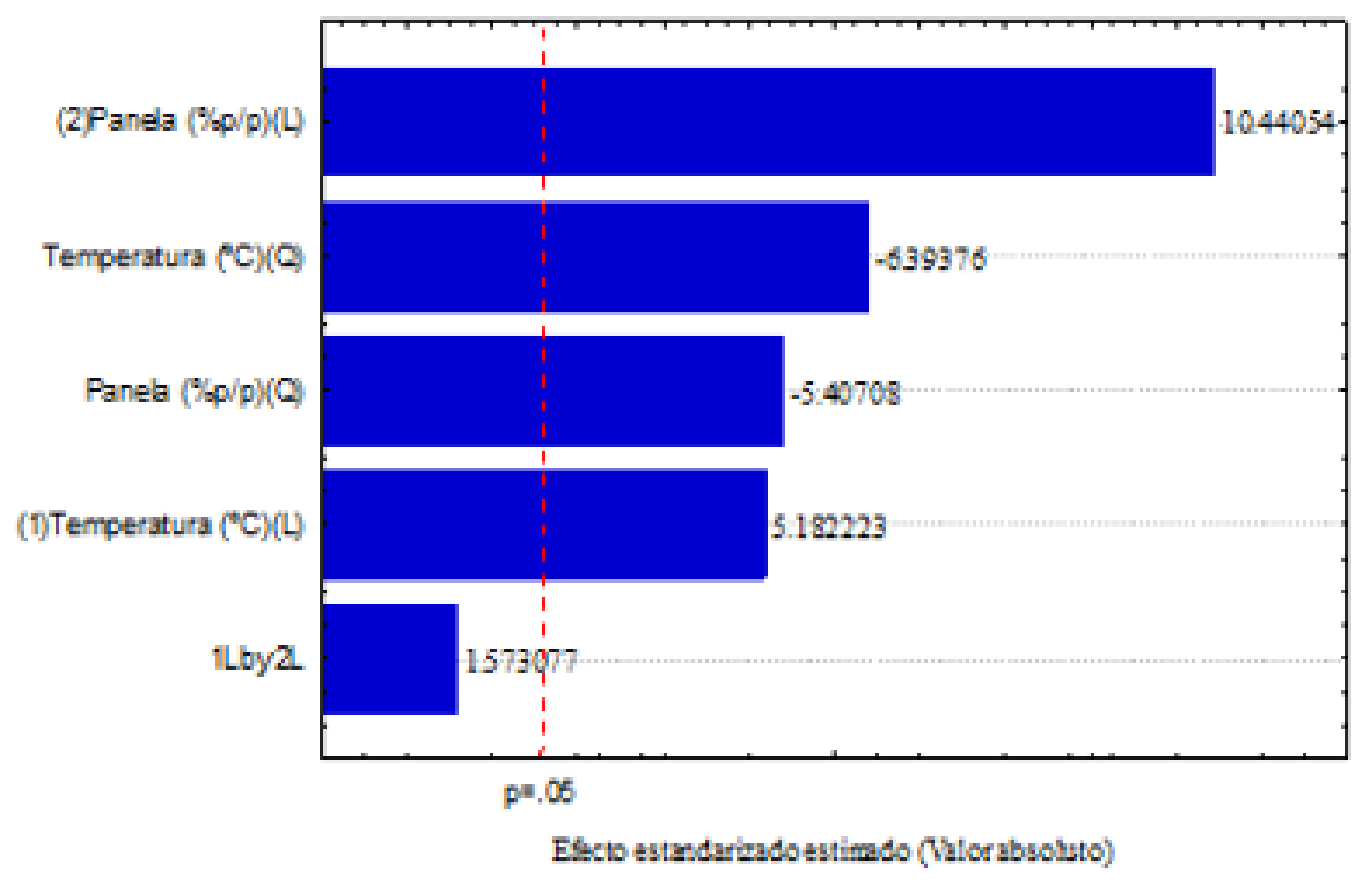

En las Tablas 8, 9, 10 y 11 se realizó un análisis de los coeficientes de regresión para evaluar su incidencia en el modelo matemático con el propósito de predecir la difusividad del agua y sólidos. En ella se observa como las variables independientes (temperatura y concentración de panela) en función lineal, cuadrática y la interacción entre ambas presentan un efecto significativo $\mathrm{p}<0.05$, tanto para el tratamiento con ultrasonido como el tratamiento testigo (sin movimiento).

\section{Tabla 8}

Coeficientes de regresión para el coeficiente de difusividad del agua $\left(D_{e, w}\right)$ en el tratamiento con ultrasonido.

\begin{tabular}{|c|c|c|c|}
\hline Factor & $\begin{array}{l}\text { Coeficientes de } \\
\text { regresión (x10- } \\
\left.{ }^{10}\right)\end{array}$ & $\begin{array}{l}\text { Error } \\
\text { estándar }\end{array}$ & Valor - p \\
\hline Mean/Interc. & -12.8278 & 0.972452 & 0.000045 \\
\hline (1) Temperatura $\left({ }^{\circ} \mathrm{C}\right)(\mathrm{L})$ & 0.4251 & 0.025624 & 0.000015 \\
\hline Temperatura $\left({ }^{\circ} \mathrm{C}\right)(\mathrm{Q})$ & -0.0060 & 0.000244 & 0.000002 \\
\hline (2) Panela $(\% p / p)(L)$ & 0.2401 & 0.025624 & 0.000233 \\
\hline Panela $(\% p / p)(Q)$ & -0.0035 & 0.000244 & 0.000029 \\
\hline $1 \mathrm{~L} \times 2 \mathrm{~L}$ & 0.0033 & 0.000290 & 0.000095 \\
\hline
\end{tabular}


Tabla 9

Coeficientes de regresión para el coeficiente de difusividad de los sólidos $\left(D_{e, s}\right)$ en el tratamiento con ultrasonido.

\begin{tabular}{lccc}
\hline \multicolumn{1}{c}{ Factor } & $\begin{array}{c}\text { Coeficientes de } \\
\text { regresión }\left(\times 10^{-10}\right)\end{array}$ & $\begin{array}{c}\text { Error } \\
\text { estándar }\end{array}$ & Valor - p \\
\hline Mean/Interc. & -7.5765 & 1.149457 & 0.001207 \\
$(1)$ Temperatura $\left({ }^{\circ} \mathrm{C}\right)(\mathrm{L})$ & 0.2109 & 0.030288 & 0.000939 \\
Temperatura $\left({ }^{\circ} \mathrm{C}\right)(\mathrm{Q})$ & -0.0016 & 0.000288 & 0.002840 \\
$(2)$ Panela $(\% p / p)(\mathrm{L})$ & 0.1796 & 0.030288 & 0.001946 \\
Panela $(\%$ p $/ \mathrm{p})(\mathrm{Q})$ & -0.0014 & 0.000288 & 0.004598 \\
$1 \mathrm{~L} \times 2 \mathrm{~L}$ & -0.0009 & 0.000343 & 0.043292 \\
\hline $\mathrm{R}^{2}=0.97441 ; \mathrm{R}^{2}$ Ajwstado $=0.94883 ;$ Media de cuadrados residual $=$ \\
0.0059325
\end{tabular}

\section{Tabla 10}

Coeficientes de regresión para la determinación del coeficiente de difusividad del agua $\left(D_{e, W}\right)$ en el tratamiento testigo (sin movimiento).

\begin{tabular}{lccc}
\hline \multicolumn{1}{c}{ Factor } & $\begin{array}{c}\text { Coeficientes de } \\
\text { regresión }\left(\mathrm{x} 10^{-10}\right)\end{array}$ & $\begin{array}{c}\text { Error } \\
\text { estándar }\end{array}$ & Valor - p \\
\hline Mean/Interc. & -4.4764 & 1.139783 & 0.011099 \\
(1) Temperatura $\left({ }^{\circ} \mathrm{C}\right)(\mathrm{L})$ & 0.1284 & 0.030033 & 0.007904 \\
Temperatura $\left({ }^{\circ} \mathrm{C}\right)(\mathrm{Q})$ & -0.0017 & 0.000286 & 0.002048 \\
(2) Panela $(\% \mathrm{p} / \mathrm{p})(\mathrm{L})$ & 0.0977 & 0.030033 & 0.022607 \\
Panela $(\%$ p $/ \mathrm{p})(\mathrm{Q})$ & -0.0011 & 0.000286 & 0.013333 \\
$1 \mathrm{~L}$ by 2L & 0.0009 & 0.000340 & 0.046397 \\
\hline $\mathrm{R}^{2}=0.98636 ; \mathrm{R}^{2}$ Ajwstado $=0.97273 ;$ Media de cuadrados residual $=$ \\
0.005833
\end{tabular}




\section{Tabla 11}

Coeficientes de regresión para la determinación del coeficiente de difusividad de los sólidos $\left(D_{e, s}\right)$ en el tratamiento testigo (sin movimiento).

\begin{tabular}{lccc}
\hline \multicolumn{1}{c}{ Factor } & $\begin{array}{c}\text { Coeficientes } \\
\text { de regresión } \\
\left(\mathrm{x} 10^{-16}\right)\end{array}$ & $\begin{array}{c}\text { Error } \\
\text { estándar }\end{array}$ & Valor - p \\
\hline Mean/Interc. & -5.5899 & 1.088624 & 0.003661 \\
(1) Temperatura $\left({ }^{\circ} \mathrm{C}\right)(\mathrm{L})$ & 0.1467 & 0.028685 & 0.003725 \\
Temperatura $\left({ }^{\circ} \mathrm{C}\right)(\mathrm{Q})$ & -0.0017 & 0.000273 & 0.001387 \\
$(2)$ Panela $(\% \mathrm{p} / \mathrm{p})(\mathrm{L})$ & 0.1353 & 0.028685 & 0.005266 \\
Panela $(\% \mathrm{p} / \mathrm{p})(\mathrm{Q})$ & -0.0015 & 0.000273 & 0.002925 \\
1L by 2L & 0.0005 & 0.000325 & 0.176511 \\
\hline $\mathrm{R}^{2}=0.97472 ; \mathrm{R}^{2}$ «jostado $=0.94945 ;$ Media de cuadrados residual $=$ \\
0.0053212
\end{tabular}

Con la determinación de los coeficientes de regresión mostrados en las Tablas 8, 9, 10 y 11, se obtuvieron los siguientes modelos matemáticos que permiten predecir la difusividad de agua y sólidos en el tratamiento usando ultrasonido y el tratamiento testigo (sin movimiento):

$$
\begin{gathered}
Y_{W U}=-12.8278 \times 10^{-10}+0.4251 \times 10^{-10} X_{1}-0.0060 \times 10^{-10} X_{1}^{2} \\
+0.2401 \times 10^{-10} X_{2}-0.0035 \times 10^{-10} X_{2}^{2}+0.0033 \times 10^{-10} X_{1} X_{2} \\
Y_{S U}=-7.5765 \times 10^{-10}+0.2109 \times 10^{-10} X_{1}-0.0016 \times 10^{-10} X_{1}^{2}+0.1796 \times 10^{-10} X_{2} \\
-0.0014 \times 10^{-10} X_{2}^{2}-0.0009 \times 10^{-10} X_{1} X_{2} \\
Y_{W T}=-4.6764 \times 10^{-10}+0.1284 \times 10^{-10} X_{1}-0.0017 \times 10^{-10} X_{1}^{2}+0.0977 \times 10^{-10} X_{2} \\
-0.0011 \times 10^{-10} X_{2}^{2}+0^{0.0009 \times 10^{-10} X_{1} X_{2}} \\
Y_{S T}=-5.5899 \times 10^{-10}+0.1467 \times 10^{-10} X_{1}-0.0017 \times 10^{-10} X_{1}^{2}+0.1353 \times 10^{-10} X_{2} \\
-0.0015 \times 10^{-10} X_{2}^{2}-0.0005 \times 10^{-10} X_{1} X_{2}
\end{gathered}
$$

Donde $\mathrm{Y}_{\mathrm{wU}}$ y $\mathrm{Y}_{\mathrm{SU}}$ constituyen los coeficientes de difusividad promedio para el agua y solutos en el tratamiento usando ultrasonido, $\mathrm{X}_{1}$ es la temperatura del medio osmótico $\left({ }^{\circ} \mathrm{C}\right)$ y $\mathrm{X}_{2}$, la concentración de panela $(\% \mathrm{p} / \mathrm{p})$; y $\mathrm{Y}_{\mathrm{wT}}$ y $\mathrm{Y}_{\mathrm{ST}}$ constituyen los coeficientes de difusividad promedio para el agua y solutos en el tratamiento sin 
ultrasonido (testigo), $\mathrm{X}_{1}$ es la temperatura del medio osmótico $\left({ }^{\circ} \mathrm{C}\right)$ y $\mathrm{X}_{2}$, la concentración de panela $(\% \mathrm{p} / \mathrm{p})$.

Para evaluar la confiabilidad con las ecuaciones obtenidas se determinó el error medio relativo tanto para el tratamiento con ultrasonido como para el tratamiento sin ultrasonido, y se llevó a cabo un ensayo extra en una disolución de panela a $50 \%(\mathrm{p} / \mathrm{p})$ y $50{ }^{\circ} \mathrm{C}$ de temperatura, calculando los coeficientes de difusividad media tanto para el agua y sólidos (Tabla 12 y 13), donde el EMR llega a un valor máximo de $3.7448 \%$ y Azuara et al. (1996) consideran que un valor máximo de $10 \%$ de error medio relativo permite que el modelo matemático tenga un buen ajuste con los datos experimentales.

\section{Tabla 12}

Error medio relativo en $D_{e, W}$ y $D_{e, s}$ experimental y teórico para la deshidratación osmótica de cubos de maca con ultrasonido (40k Hz).

\begin{tabular}{lccc}
\hline Componente & $\begin{array}{c}\text { Experimental } \\
(\mathbf{x 1 0 - 1 0})\end{array}$ & $\begin{array}{c}\text { Térico } \\
\left(\mathbf{x 1 0} 0^{-10}\right)^{*}\end{array}$ & EMR (\%) \\
\hline Agua & 4.9322 & 4.7475 & 3.7448 \\
Sólidos & 2.1985 & 2.2136 & -0.6865 \\
\hline
\end{tabular}

*Hace referencia a los modelos matemáticos obtenidos ( $\left.\mathrm{Y}_{\mathrm{wu}} \mathrm{y} \mathrm{Y}_{\mathrm{su}}\right)$

\section{Tabla 13}

Error medio relativo en $D_{e, W}$ y $D_{e, s}$ experimental $\left(50^{\circ} \mathrm{C}\right.$ y $\left.50 \% \mathrm{p} / \mathrm{p}\right)$ y teórico para la osmodeshidratación de cubos de maca testigo (sin movimiento).

\begin{tabular}{lccc}
\hline Componente & $\begin{array}{c}\text { Experimental } \\
\left(\mathbf{x} 0^{-10}\right)\end{array}$ & $\begin{array}{c}\text { Teórico } \\
\left(\mathbf{x 1 0} 0^{-10}\right)^{*}\end{array}$ & EMR (\%) \\
\hline Agua & 2.0786 & 2.1993 & -5.8044 \\
Sólidos & 1.7601 & 1.7314 & 1.6328 \\
\hline
\end{tabular}

*Hace referencia a los modelos matemáticos obtenidos ( $\mathrm{Y}_{\mathrm{WT}}$ y $\left.\mathrm{Y}_{\mathrm{ST}}\right)$

Las Figuras 7 y 8 muestran las curvas de contorno para el coeficiente de difusividad del agua y sólidos por acción de temperatura y concentración de panela en el tratamiento con ultrasonido $(40 \mathrm{kHz})$. En ella se observa que entre la temperatura de $48-54^{\circ} \mathrm{C}$ y $54-61 \% \mathrm{p} / \mathrm{p}$ se obtiene los mayores valores de coeficiente de difusividad, y en las figuras 9 y 10 se observa las gráficas de contorno en la osmodeshidratación de cubos de maca sin movimiento (testigo) para el coeficiente de difusividad de agua y de sólidos respectivamente. En ellas se observa que entre las temperaturas de $46-52^{\circ} \mathrm{C}$ y concentración de panela de $50-56 \%$ p/p se obtiene los mayores valores de coeficiente de difusividad para el agua y sólidos. 


\section{Figura 7}

Gráfico de contorno del efecto de la temperatura y concentración de panela en el coeficiente de difusividad del agua $\left.\left(D_{e, w}\right)\left(\mathrm{m}^{2} / \mathrm{s} \times 10^{10}\right)\right)$ en la osmodeshidratación de cubos de maca con ultrasonido.

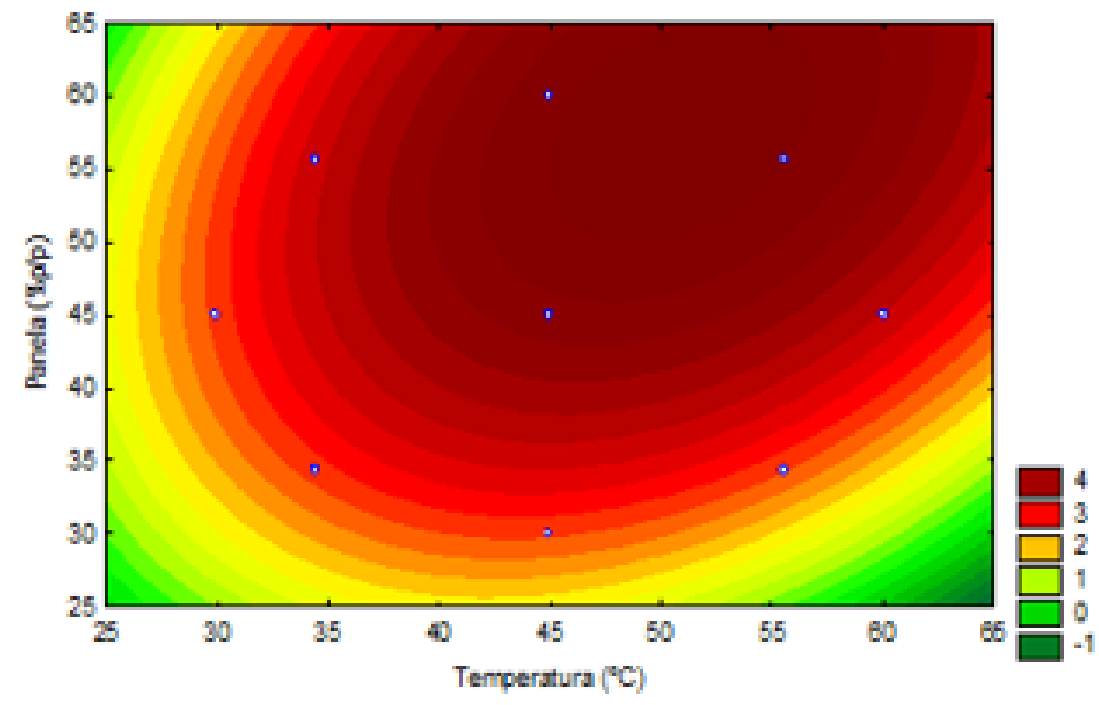

\section{Figura 8}

Gráfico de contorno del efecto de la temperatura y concentración de panela en el coeficiente de difusividad de los sólidos $\left(D_{e, s}\right)\left(\mathrm{m}^{2} / \mathrm{s}_{X} 10^{10}\right)$ en la osmodeshidratación de cubos de maca con ultrasonido.

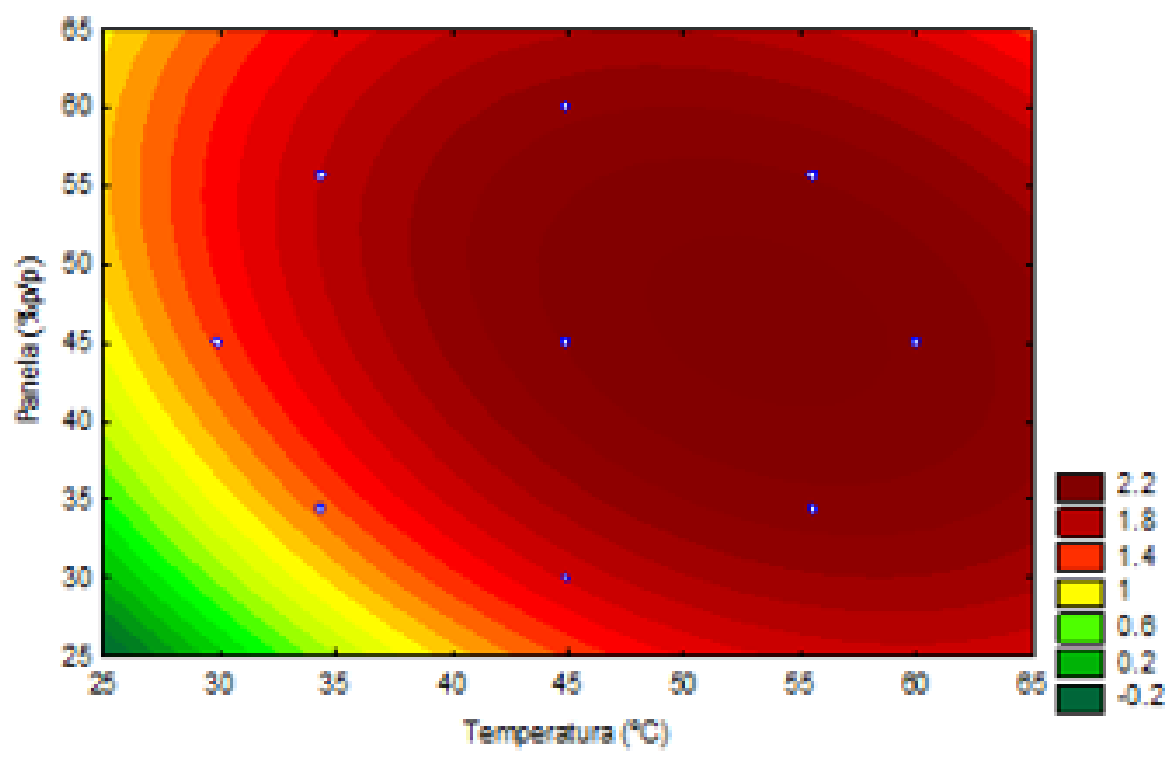




\section{Figura 9}

Gráfico de contorno del efecto de las variables independientes sobre el coeficiente de difusividad del agua $\left.D_{e, W}\left(\mathrm{~m}^{2} / \mathrm{s} \times 10^{10}\right)\right)$ en la osmodeshidratación de cubos de maca sin movimiento (testigo).

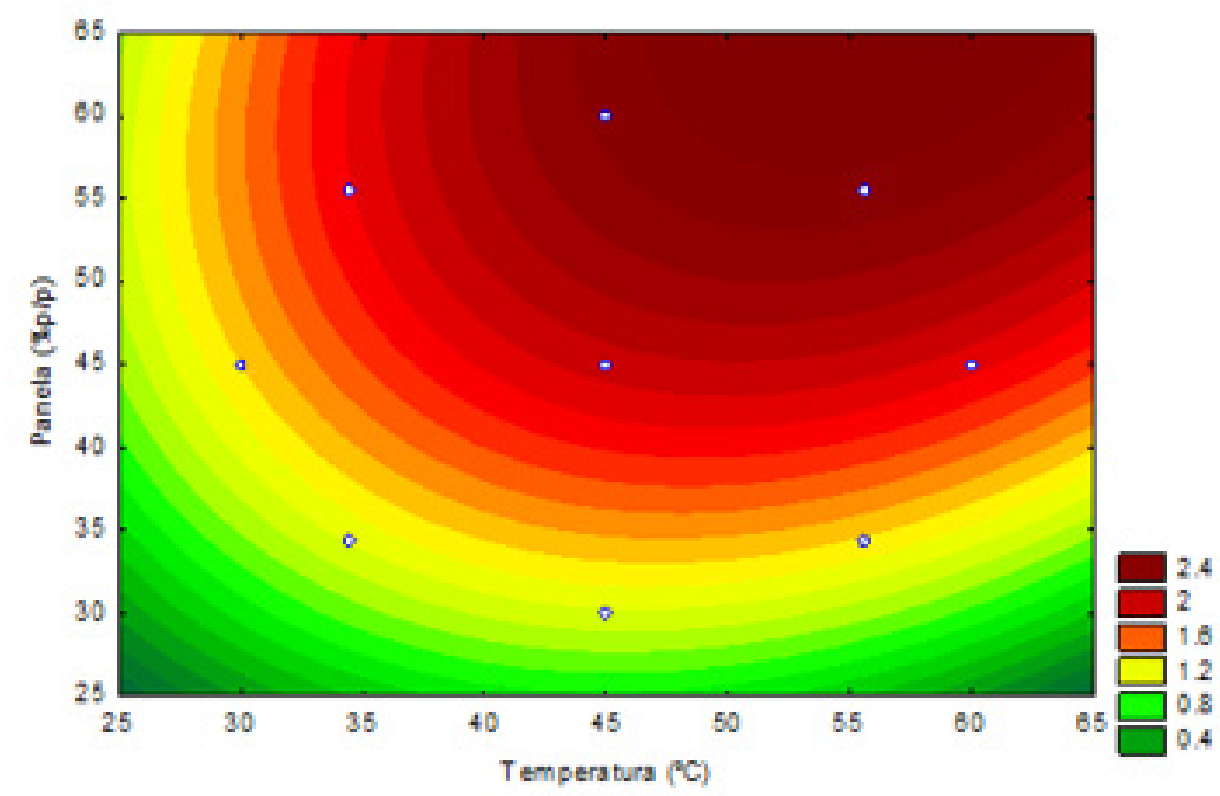

\section{Figura 10}

Gráfico de contorno del efecto de las variables independientes sobre el coeficiente de difusividad efectiva de solutos $D_{e, s}\left(\mathrm{~m}^{2} / \mathrm{s} \times 10^{10}\right)$ en la osmodeshidratación de cubos de maca sin movimiento (testigo).

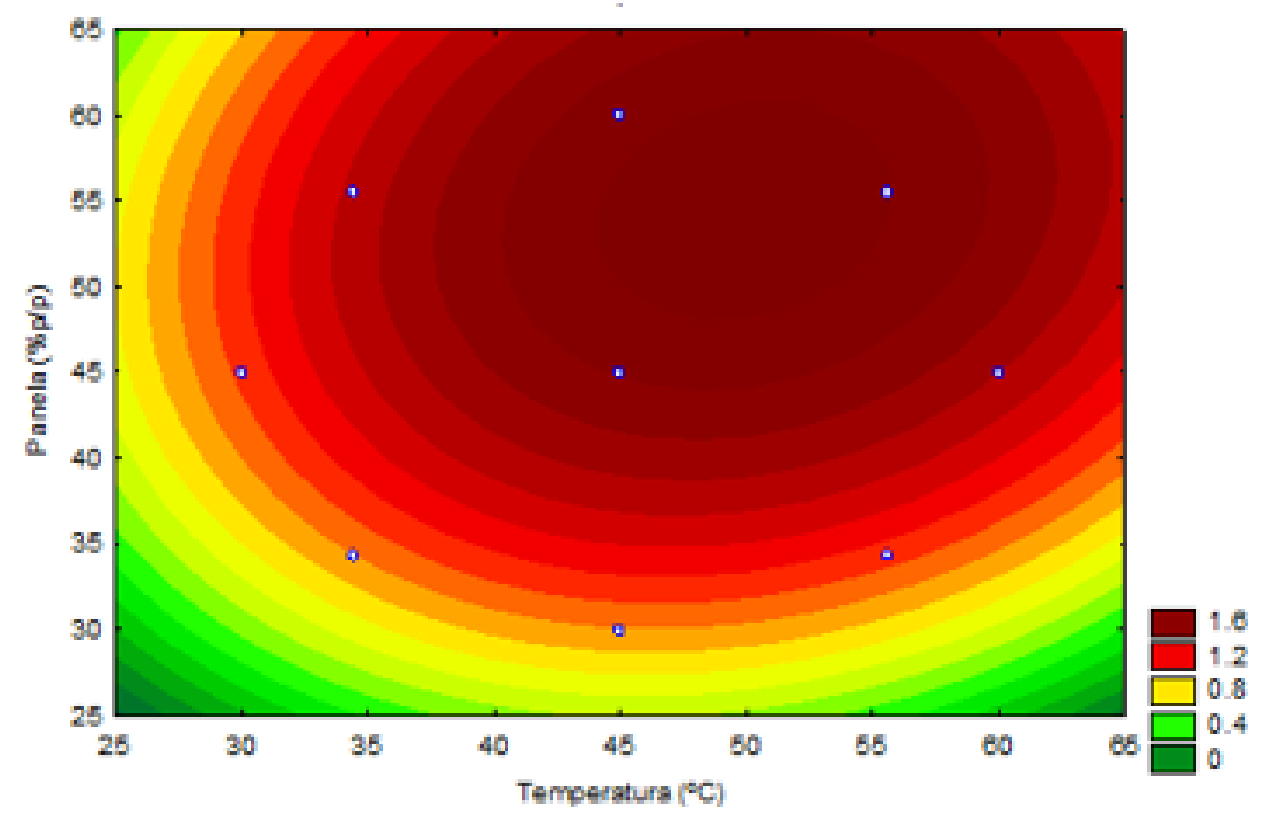




\section{Conclusiones}

La caracterización fisicoquímica de la maca (Lepidium meyenii) reporta un contenido de sólidos de $20.69 \%$, una concentración de minerales expresado como contenido de cenizas de $2.41 \mathrm{~g} / 100 \mathrm{~g}, 6.67^{\circ}$ Brix de sólidos solubles, $2.50 \%$ de azúcares reductores y una capacidad antioxidante de $19.81 \%$.

La cinética de osmodeshidratación de maca permitió identificar que la pérdida de agua y ganancia de sólidos se da mayoritariamente en las primeras dos horas de tratamiento, llegando a perder luego de siete horas $53.57-24.56 \%$ de agua en los ensayos con ultrasonido y testigo respectivamente; en tanto que para ganancia de sólidos en los mismos ensayos fue de $18.61-9.01 \%$ respectivamente.

Los coeficientes de difusividad efectiva fueron mayores en el tratamiento usando ultrasonido tanto en la pérdida de agua como para ganancia de sólidos. De tal manera que con el uso de ultrasonido se obtuvo coeficientes de difusividad entre $2.5142-4.7412 \mathrm{x}$ $10^{-10} \mathrm{~m}^{2} / \mathrm{s}$ y $1.1643-2.2087 \times 10^{-10} \mathrm{~m}^{2} / \mathrm{s}$ para el agua y sólidos respectivamente. En tanto que para el tratamiento sin ultrasonido (testigo) se obtuvo coeficientes de difusividad para pérdida de agua entre $1.1011-2.35 \times 10^{-10} \mathrm{~m}^{2} / \mathrm{s}$ y $0.9016-1.7021 \times 10^{-10} \mathrm{~m}^{2} / \mathrm{s}$ para ganancia de solutos.

El análisis de varianza muestra un efecto significativo $(\mathrm{p}>0.05)$ por parte de las variables independientes (temperatura y concentración de panela) en forma, lineal y cuadrática, sobre los factores de difusividad para el agua y solutos con ultrasonido y testigo.

En todos los ensayos estudiados, el uso de ultrasonido incrementó la pérdida de agua como ganancia de sólidos, y con ello los coeficientes de difusividad al ser comparados con los ensayos testigo (sin ultrasonido). De igual manera, el incremento de temperatura y concentración de la disolución de panela favorece eliminación de agua y ganancia de sólidos.

El tratamiento testigo tuvo los mayores valores de coeficiente de difusividad a concentraciones de panela entre $50-56 \% \mathrm{p} / \mathrm{p}$ y un intervalo de temperatura entre 46-52 ${ }^{\circ} \mathrm{C}$. En tanto que con el uso de ultrasonido $(40 \mathrm{kHz})$ sus valores máximos de difusividad se lograron a concentraciones de panela de $54-61 \% \mathrm{p} / \mathrm{p}$ y temperaturas de $48-54{ }^{\circ} \mathrm{C}$.

\section{Declaración de conflicto de intereses.}

Los autores declaran que el presente manuscrito no presenta ningún tipo de conflicto de intereses. 


\section{Referencias}

Azuara, E.; Beristain, C.; Gutiérrez, G. (2002). Osmotic dehydration of apples by immersion in concentrated sucrose/maltodextrin solutions. Journal of Food Processing Preservation, 26: 295-306. https://doi.org/10.1111/j.1745-4549.2002. $\underline{\text { tb00486.x }}$

Azuara, E.; Beristain, C.; Gutiérrez, G. (1998). A method for continuous kinetic evaluation of osmotic dehydration. LWT - Food Science and Technology, 31: 317-321. https://doi.org/10.1006/fstl.1997.0364

Azuara, E.; García, H.; Beristain, C. (1996). Effect of the centrifugal force on osmotic dehydration of potatoes and apples. Food Research International, 29(2): 195-199. https://doi.org/10.1016/0963-9969(96)00033-6

Barbosa, G.; Vega, H. (2000). Deshidratación de Alimentos, Zaragoza - España, Editorial Acribia S.A, pp. 235-253.

Bocanegra, J.; Espinosa, L. (2013). Parámetros de diseño de sistema de osmodeshidratación del yacón a partir de sus parámetros de proceso. Tesis de pregrado. Universidad Nacional de Trujillo. Trujillo, Perú.

Brand-Williams, W.; Cuvelier, M.; Berset, C. (1995). Use of free radical method to evaluate antioxidant activity. Lebensmittel-Wissenschaft an Technologie, 22: $25-30$.

Centro Nacional de Alimentación y Nutrición (2017). Tablas Peruanas de composición de alimentos. Tubérculos, raíces y derivados. Maca silvestre, pág. 66-67. https://doi.org/10.1016/S0023-6438(95)80008-5

Damodaran, S; Parkin, K. (2017). Fennema's Food Chemistry. Fifth Edition. CRC Press. USA.

Dane, C. (2015). 3er Censo Nacional Agropecuario 2014: Inventario Agropecuario en las Unidades de Producción Agropecuaria (UPA). https://www.dane.gov.co/files/ CensoAgropecuario/entrega-definitiva/Boletin-9 cultivos/9-Boletin.pdf

Dermesonlouoglou, E.; Giannakourou, M.; Taoukis, P. (2007). Stability of dehydrofrozen tomatoes pretreated with alternative osmotic solutes. Journal of Food Engineering, 78, 272 - 280. https://doi.org/10.1016/j.jfoodeng.2005.09.026

FAO (Food and Agriculture Organization of the United Nations). (1987). Manual para el mejoramiento del manejo poscosecha de frutas y hortalizas Parte I, Tecnología Poscosecha 6: 1-100. http://www.fao.org/docrep/x5055s/x5055S00.htm\# Contentsde

Gonzales, F. (2006). Maca de la tradición a la ciencia. CONCYTEC: Universidad Peruana Cayetano Heredia. 
ISO 638. (2008). International Organization for Standardization. Method for determination of dry matter content.

ISO 776. (1982). International Organization for Standardization. Method for determination of acid-insoluble ash of pulp.

Lemus, R; Vega, A; Zura, L; Ah-Hen, K. (2012). Stevia rebaudiana Bertoni, source of a high-potency natural sweetener: A comprehensive review on the biochemical, nutritional and functional aspects. Food Chemistry 132: 1121 - 1132. https://doi.org/10.1016/j.foodchem.2011.11.140

Montgomery, D. (2017). Desing and analysis of experiments. Ninth Edition. John Wiley \& Sons, USA.

Nahimana, H.; Zhang, M.; Mujumdar, A.; Díaz, Z. (2011). Mass transfer modeling and shrinkage consideration during osmotic dehydration of fruits and vegetables. Food Reviews International, 27(4), 331 - 356. https://doi. org/10.1080/87559129.2010.518298

NMX-F-103. (1982). Normas Mexicanas. Dirección General de Normas. Determinación de Grados Brix.

NMX-F-312. (1978). Normas Mexicanas. Dirección General de Normas. Determinación de azúcares reductores en alimentos.

Obregón, L. (1998). Maca, planta medicinal y nutrientes del Perú. Instituto de Fitoterapia Americano. Lima, Perú.

Peña, A. (2019). Efecto de la temperatura y concentración de panela en la cinética de osmodeshidratación de betarraga (Beta vulgaris L.) asistido con ultrasonido. Tesis de pregrado. Universidad Nacional de Trujillo. Trujillo, Perú.

Raoult, L.; Wack, A. (1992). Recient advance in the osmotic dehydration of food. Food Science and Technology, (05):255-260. https://doi.org/10.1016/09 24-2244(94)90018-3

Rodríguez, E. (2019). Efecto de la temperatura y concentración de panela en la osmodeshidratación de camote (Ipomoea batata L.) asistido por ultrasonido y agitación. Tesis de pregrado. Universidad Nacional de Trujillo. Trujillo, Perú.

Suca, C.; Suca, G. (2010). Deshidratación osmótica de alimentos. Boletín de divulgación Tecnológica Agroindustrial. Enero. 1(1).

Valerio, L.; Gonzales, F. (2005). Toxicological aspects of south american herbs: Uncaria tomentosa (Cat's claw) and Lepidium meyenii (Maca). A Critical Synopsis. Toxicological Review, 24: 11-35. DOI: $10.2165 / 0013970$ 9-200524010-00002 
Yao, L.; Zhao, Q.; Xiao, J.; Sun, J.; Yuan, X.; Zhao, B. (2012). Composition and antioxidant activity of the polysaccharides from cultivated Saussurea involucrata. International Journal of Biological Macromolecules. 50: 849 - 853. https://doi.org/10.1016/j.ijbiomac.2011.11.012 